\title{
Allergic bronchopulmonary aspergillosis in cystic fibrosis. A European epidemiological study
}

\author{
G. Mastella*, M. Rainisio ${ }^{\#}$, H.K. Harms ${ }^{+}$, M.E. Hodson**, C. Koch ${ }^{\# \#, ~ J . ~ N a v a r r o ~}{ }^{++}$, B. Strandvik***, \\ S.G. McKenzie ${ }^{\# \#}$ on behalf of the investigators of the Epidemiologic Registry of Cystic Fibrosis
}

\begin{abstract}
Allergic bronchopulmonary aspergillosis in cystic fibrosis. A European epidemiological study. G. Mastella, M. Rainisio, H.K. Harms, M.E. Hodson, C. Koch, J. Navarro, B. Strandvik, S.G. McKenzie on behalf of the investigators of the Epidemiologic Registry of Cystic Fibrosis. C ERS Journals Ltd 2000.

ABSTRACT: Allergic bronchopulmonary aspergillosis (ABPA) is a disease resulting from a hypersensitivity response to Aspergillus fumigatus, although the pathogenesis of the disease is unknown and its prevalence in cystic fibrosis (CF) is still poorly defined.

Data from the Epidemiologic Registry of Cystic Fibrosis (ERCF) on 12,447 CF patients gathered from $224 \mathrm{CF}$ centres in nine European countries were analysed. The ERCF definition of ABPA diagnosis is a positive skin test and serum precipitins to $A$. fumigatus, together with serum immunoglobulin (Ig)E levels $>1,000 \mathrm{U} \cdot \mathrm{mL}^{-1}$ and additional clinical or laboratory parameters.

The overall prevalence of ABPA in the ERCF population was $7.8 \%$ (range: $2.1 \%$ in Sweden to $13.6 \%$ in Belgium). Prevalence was low $<6$ yrs of age but was almost constant $\sim 10 \%$ thereafter. No sex differences were observed. ABPA affected $8.0 \%$ of patients with a $\Delta$ F508/ $\Delta$ F508 genotype and 5-6\% with $\Delta$ F508/G551D, $\Delta$ F508/G542X and $\Delta \mathrm{F508} / \mathrm{N} 1303 \mathrm{~K}$ genotypes. ABPA patients presented a lower forced expiratory volume in one second (FEV1) than those without ABPA at any age and the prevalence ranged from $6.6 \%$ in patients with FEV1 $\geq 20-12.9 \%$ in those with FEV1 $<40 \%$. ABPA was associated with higher rates of microbial colonization, pneumothorax and massive haemoptysis, and with higher IgG serum levels and poorer nutritional status. A mixed model regression analysis of lung function showed that FEV1 decline during the follow-up period was not substantially different in ABPA patients compared with non-ABPA patients for any subgroups based on age or disease severity at enrolment.

To conclude, allergic bronchopulmonary aspergillosis is a frequent complication in cystic fibrosis patients, particularly after the age of $6 \mathrm{yrs}$, and it is generally associated with a poorer clinical condition. However, any clear independent influence of allergic bronchopulmonary aspergillosis on the rate of lung function decline in the short term was not shown.
\end{abstract}

Eur Respir J 2000; 16: 464-471.

Allergic bronchopulmonary aspergillosis (ABPA) is a disease resulting from a hypersensitivity response to $A s$ pergillus fumigatus, an ubiquitous fungal pathogen that can colonise the bronchial tree [1]. In reality, although $A$. fumigatus is the primary causative pathogen in ABPA, the disease can be caused by several other members of the Aspergillus spp, including A. flavus, A. nidulans, A. niger, as well as other fungi [2].

Patients with asthma are predisposed to developing ABPA, with a prevalence of $1-2 \%[3]$, and the pulmonary status of cystic fibrosis (CF) patients represents a favourable environment for the development of ABPA. The pathogenesis of the disease is unknown, although it is postulated that $A$. fumigatus spores are inhaled, trapped in the mucus of the large segmental bronchi, and subsequently germinate to form hyphae, which release antigens to elicit an immune response [3].
*Dept of Pulmonary and Digestive Diseases of Developmental Age, Cystic Fibrosis Center, Verona, Italy. "Statistics for Research SFR Ltd, Basel, Switzerland. ${ }^{+}$Children's Hospital, Munich University, Munich, Germany. ${ }^{* *}$ Cystic Fibrosis Dept, Royal Brompton National Heart \& Lung Hospital, London, UK. "\# Dept of Paediatrics, Rigshospitalet, University Hospital, Copenhagen, Denmark. ${ }^{++}$Dept of Gastroenterology, Robert Debré Hospital, Paris, France. $* * *$ Dept of Pediatrics, OestraUniversity Hospital, Göteborg, Sweden. "\#\# Pharmaceuticals Division, F. Hoffmann-La Roche Ltd, Basel, Switzerland.

Correspondence: G. Mastella, Cystic Fibrosis Center Ospedale Civile Maggiore Piazzale Stefani 137126 Verona Italy Fax: 39458072042

Keywords: Allergic bronchopulmonary aspergillosis, cystic fibrosis, lung function, mixed model

Received: June 301999

Accepted after revision March 192000

The Epidemiologic Registry of Cystic Fibrosis (ERCF) Advisory Board, an independent team of academics who manage the ERCF with complete editorial freedom, would-like to acknowledge financial support from F. Hoffmann-La Roche, Basel, Switzerland for funding the ERCF.
The hypersensitivity reaction is characterized by the production of immunoglobulin (Ig) $\mathrm{G}$ and $\mathrm{IgE}$ antibodies specific for A. fumigatus, as well as a rise in total $\operatorname{IgE}[4,5]$. This reaction, in combination with the generation of cytotoxic metabolites such as proteolytic enzymes by $A$. fumigatus, causes local immunosuppression, inhibition of phagocytosis and epithelial cell detachment [6]. Together these effects enhance A. fumigatus colonization and facilitate its persistence in the respiratory tract, encouraging the accumulation of pulmonary infiltrates, tissue damage, and eventually destruction of lung tissue [3-6].

The prevalence of ABPA in CF is still poorly defined. Available literature suggests a wide range of frequencies from $0-25 \%$ [7-14], which may in part be due to the use of different diagnostic criteria. Validated criteria for the diagnosis of ABPA have still not been established in CF and the situation has been critically discussed in a recent 
epidemiological study [12]. In CF, diagnosis relies principally on immunological evidence (in particular a positive, immediate skin reactivity to A. fumigatus and on the in vitro demonstration of specific serum $\mathrm{IgE}$, IgG and precipitating antibodies). Asthma, total serum $\operatorname{IgE}$ and peripheral eosinophilia are important additional criteria. Pulmonary infiltrates and central bronchiectasis on chest radiographs, usually suggestive of ABPA [1, 15], are commonly encountered in $\mathrm{CF}$ as a result of chronic bacterial infection and should be viewed with caution as selective criteria for diagnosis. The recommended combination of immunological evidence and clinical manifestations is important. Positive immune parameters and elevated total serum $\operatorname{IgE}$ levels in a patient experiencing increased cough or wheezing, pulmonary infiltrates, or a decrease in pulmonary function that are unresponsive to aggressive therapy (increased antibiotics and bronchodilators) may suggest a diagnosis of ABPA [13, 14, 16, 20]. In reality, sensitization to $A$. fumigatus is very common in $\mathrm{CF}$, occurring in $\geq 50 \%$ of patients $[10,13,14,21-23]$. In a prospective study of 222 consecutive CF patients $>6$ yrs of age, $56 \%$ were sensitized to A. fumigatus and $23 \%$ of these patients had ABPA [10]. In contrast, in a study of 51 adult CF patients, the immune response to A. fumigatus was present in $66 \%$ of patients, but only one patient was considered to have ABPA [12]. Therefore, A. fumigatus sensitization, in the absence of clinical features of ABPA, does not suggest an ABPA diagnosis.

One of the most relevant problems is the contribution of ABPA to disease progression in CF. This is a poorly documented and little understood issue for which no epidemiological studies have been performed to date. In spite of this, ABPA is considered to be a serious complication in patients with $\mathrm{CF}[8,16,17-19]$.

The data collated from a large European CF population (the Epidemiologic Registry of Cystic Fibrosis (ERCF)) permitted the present study. The main aim of this study was to establish the prevalence of ABPA in CF. Secondary aims were to describe the clinical conditions that can be associated with ABPA and to explore the possible influence of ABPA on the progression of lung disease. In the ERCF the diagnosis of ABPA is defined according to the above criteria and other recommendations.

\section{Patients and methods}

\section{Data ascertainment and quality control}

The ERCF is an international observational database initiated in Europe by F. Hoffmann-La Roche Ltd in 1994. Countries were eligible to participate if the local company affiliate had sufficient available manpower and financial resources to support-initiation of the Registry. Clinics in these countries were eligible to participate if they delivered specialized care to $>10 \mathrm{CF}$ patients and were willing to submit defined clinical data collected from each of their patients at normal clinic visits.

Initially, data were collected on paper forms that were sent to Quintiles GmbH (Neu Isenburg, Germany) for data entry, quality control and assignment of preferred terms (coding) of CFTR mutations, medications, adverse events and causes of death. Quintiles subjected the data to $>400$ context and consistency checks and issued queries to the submitting centre where necessary to clarify inconsistencies. In 1997 a transition was made to remote data entry (RDE) using Internet technology. The data entry forms were recreated in Java script and the pre-existing quality assurance checks (including longitudinal checks) and most of the coding were built into the RDE system to ensure high data quality. Participating centres were given controlled access to the data entry screens at www.ercf.org and the locally entered data were submitted in encrypted form to Spider Network AG (Otelfingen, Switzerland) for subsequent transfer to Quintiles.

Data analysis was under the control of an Advisory Board comprised of the authors of this manuscript and at no time were personal patient data available to HoffmannLa Roche Ltd.

\section{Patients and cystic fibrosis centres}

A total of $12,447 \mathrm{CF}$ patients were enrolled in the ERCF by 13 December 1997 (5,967 females (48\%), median age 12 yrs, age range from 0-62 yrs). Patients were enrolled in the ERCF from $224 \mathrm{CF}$ centres in nine European countries (Austria, Belgium, Denmark, France, Germany, Ireland, Sweden, The Netherlands and the UK). Seventy-three centres enrolled $\geq 50$ patients.

\section{Allergic bronchopulmonary aspergillosis diagnosis}

The ERCF requires that the diagnosis of ABPA be reported, as defined by the following criteria: a positive immediate (type 1) skin test reaction to $A$. fumigatus, multiple serum precipitins to A. fumigatus, a total serum IgE level $>1,000 \mathrm{U} \cdot \mathrm{mL}^{-1}$, and a physician's suspicion that the patient has ABPA, based in part on one or more of the following: reversible bronchoconstriction or asthma, pulmonary infiltrates, elevated serum IgE and/or IgG specific for A fumigatus, peripheral eosinophilia $\left(>1,000 \mathrm{~mL}^{-1}\right)$, recovery of A. fumigatus from sputum or hyphae present on smear, and response to steroids. In the absence of validated diagnostic criteria in CF, the physician's suspicion of ABPA based on multiple clinical, radiological and additional immunological conditions was considered a reasonable compromise to overcome the problem that the usual clinical and radiological criteria of ABPA are not selective in CF [2, 3].

\section{Data handling criteria}

ABPA was studied in relation to the specific care centre, country, sex, CFTR genotype, age at enrolment, forced expiratory volume in one second (FEV1) and forced vital capacity (FVC) at enrolment and during the follow-up period, as well as body weight, serum IgG values, bacterial isolates from sputum, pulmonary complications (massive haemoptysis, pneumothorax, lung transplantation) and routine respiratory therapies, including antibiotics. The routine respiratory therapies were the usual therapeutic strategies adopted for the individual patients during the observation period, as recorded by the participating centres.

All data entered in the ERCF database from 28 September 1993 to 13 December 1997 were used for this 
analysis, independent of the patients' clinical status at the time of each reported visit. The ERCF population was stratified by the absence/presence of ABPA. The ABPA positive subpopulation was selected using information from all visits. Individual events and medical conditions were considered present if they were recorded at least once during the observation period for each patient.

Spirometric tests, performed using either a bell-spirometer or a pneumotachograph following the ERS criteria, were recorded at follow-up visits, whether patients were in stable clinical situation or not. FEV1 and FVC were calculated as percentages of the predicted values, according to KNUDSON et al. [24]. This standardization algorithm was adopted to make the results comparable with those of the United States Cystic Fibrosis Foundation and other epidemiologic registries which are in progress in USA and Canada [12]. The initial FEV1 was used to determine the severity of the disease in patients $\geq 6$ yrs of age.

The valid ERCF population was defined according to the particular analysis performed. For the genotype correlation, only patients with a known genotype were analysed; for the microbiological analysis, only patients with at least one valid sputum culture were included; for lung function analysis, the availability of FEV1 or FVC values in patients $\leq 6$ yrs of age were critical, however, valid lung function data were only available in a few patients. For $\mathrm{IgG}$, the mean of all valid values for a subject was used in the analysis. For the weight $\mathrm{z}$-scores (the standardized weight for age and sex: the z-score corresponds to the standard deviation from the mean of the reference population), the age in months was completed using the date of each visit and the date of birth, and was used together with actual weight to derive the z-score from sex-specific reference values published by the World Health Organization (WHO) [25]. The mean value was computed using all valid values for a subject. The antibiotic treatment analysis was performed by classifying the antibiotic regimens on the basis of their intensity, i.e. in order of decreasing intensity: prophylaxis, continuous use; prophylaxis, intermittent use; only for respiratory tract infection exacerbations. Any patient with more than one antibiotic regimen was considered to belong to the most intense of the reported classes. Anti-Aspergillus antibiotics were itraconazole or amphotericin.

\section{Statistical analysis}

The mixed model analysis [26, 27], which has been used to study the influence of ABPA on decline of lung function, is an extension of multiple regression analysis. Although it is a complex computation, it enables the estimation of individual and population regression parameters even in the presence of unevenly spaced repeated measures.

The intention was to model the FEV1 percent predicted value at a given age using an initial severity score (severe: FEV1 <40\%; moderate: FEV1 40-69\%; mild: FEV1 $\geq 70 \%$ ), age groups at the start of the observation period ( $<6$ yrs, 6-12 yrs, 13-17 yrs or $\geq 18$ yrs), age at the time of observation and presence/absence of ABPA as fixed effects, and age and an intercept (the intersection point between the regression line and the y-axis) as random effects. All ERCF patients with at least two FEV1 measurements after the initial one (which was not used in the analysis) were included. However, due to the very large number of observations, the full database could not be analysed and the model was fitted independently to twelve subpopulations, defined by the initial severity and age subgroups. This involved the presence/absence of ABPA and age (as fixed effects), and an intercept and age (as random effects) only. The goal of this analysis was purely descriptive, and the results of statistical tests are reported only for formal reasons. In fact, due to the unusually large number of patients in each of the analyses, statistical significance can be reached in cases where the results are of no clinical relevance.

Duration of follow-up for the mixed model analysis ranged from 1-45 months, with a median of 25 months for both the ABPA and non-ABPA groups. The number of FEV1 measurements per person ranged from $3-45$, with median values of 7 and 6 for the ABPA and non-ABPA groups, respectively.

\section{Results}

\section{Allergic bronchopulmonary aspergillosis prevalence}

The diagnosis of ABPA was reported for 967 of the 12,447 CF patients; an overall ABPA prevalence of $7.8 \%$ (95\% confidence interval (CI) 7.3-8.3). However, prevalence varied greatly between countries and individual centres. Prevalence of ABPA ranged from $2.1 \%$ in Sweden to $13.6 \%$ in Belgium (table 1), and from $0-27 \%$ for the bigger centres (i.e. those with $\geq 50$ patients; $n=73$ ). Similar variability was also apparent within individual countries.

ABPA was almost equally prevalent in males (509/6480, $7.9 \%$ ) and females $(458 / 5967,7.7 \%)$. It was little represented in patients $<6$ yrs old, but was reported in $\sim 10 \%$ of older patients $(<6$ yrs 56/3150,1.7\%; 6-12 yrs 320/ $3223,9.0 \%$; $13-18$ yrs $237 / 1879,11.2 \%$; $\geq 18$ yrs $354 /$ $3228,9.9 \%$ ). ABPA did not show a significant association with a particular CFTR genotype. Of the patients in the ERCF who had a known genotype $(n=9584)$, a comparable prevalence of ABPA was demonstrated in individuals with all of the most common genotypes $(\Delta \mathrm{F} 508 / \Delta \mathrm{F} 508,412 /$ $4765,8.0 \%$; $\Delta \mathrm{F} 508 / \mathrm{G} 551 \mathrm{D}, 11 / 205,5.1 \% ; \Delta \mathrm{F} 508 / \mathrm{G} 542 \mathrm{X}$, $11 / 185,5.6 \% ; \Delta \mathrm{F} 508 / \mathrm{N} 1303 \mathrm{~K}, 7 / 107,6.1 \%$; $\Delta \mathrm{F} 508 /$ other, 216/2627, 7.6\%; other/other, 66/972, 6.4\%).

\section{Allergic bronchopulmonary aspergillosis associated with clinical conditions}

Patient body weight, calculated in terms of z-score, was generally lower in ABPA patients than in those without ABPA (mean values of $-0.9 \pm 1.2$ and $-0.7 \pm 1.4$, respectively). In addition, ABPA patients showed elevated serum IgG levels compared with those without ABPA (mean values of $14.3 \pm 6.5 \mathrm{~g} \cdot \mathrm{L}^{-1}(\mathrm{n}=626)$ and $12.4 \pm 6.3 \mathrm{~g} \cdot \mathrm{L}^{-1}$ $(\mathrm{n}=5693)$, respectively).

Patients with ABPA had, on average, a 10\% lower FEV1 value than those without (table 2). A similar difference was found for FVC (mean values of $75.5 \%(n=906)$ and $81.8 \%(\mathrm{n}=8721)$, respectively). FEV1 percentage values were lower in the ABPA patients of all age classes compared with those without ABPA. Stratification of the patients by age and FEV1 at enrolment demonstrated an ABPA prevalence in individuals with severe or moderate 
Table 1. - Prevalence of allergic bronchopulmonary aspergillosis (ABPA) by country

\begin{tabular}{lrcc}
\hline Country & CF population & ABPA\% & $95 \%$ CI \\
\hline Austria & 169 & 5.3 & $2.5-9.9$ \\
Belgium & 44 & 13.6 & $5.2-27.4$ \\
Denmark & 320 & 3.4 & $1.7-6.1$ \\
France & 2935 & 7.4 & $6.5-8.4$ \\
Germany & 3070 & 11.5 & $10.4-12.7$ \\
Ireland & 621 & 11.0 & $8.6-13.7$ \\
Netherlands & 353 & 6.8 & $4.4-9.9$ \\
Sweden & 424 & 2.1 & $1.0-4.0$ \\
United Kingdom & 4511 & 6.0 & $5.3-6.7$ \\
All countries & 12447 & 7.8 & $7.3-8.3$ \\
\hline
\end{tabular}

CF: cystic fibrosis; 95\% CI: 95\% confidence interval.

lung disease (FEV $1<40 \%$ and $40-70 \%$, respectively), which was twice that observed in patients with mild lung disease (FEV1 $\geq 70 \%$ ). Furthermore, adolescents (patients aged 13-17 yrs) had a slightly higher; although not significant prevalence of ABPA overall and in the severe and mild classes, while patients aged 6-12 yrs had the highest prevalence among the class with moderate lung disease.

The association of specific microbial infections with ABPA is shown in table 3 . Notably, only $45 \%$ of patients with ABPA reported positive cultures for A. fumigatus, while ABPA prevalence among the A. fumigatus positives was $429 / 2193(19.6 \%)$ compared with an overall prevalence of $8.1 \%$ in the patient population with microbiological data available. The colonization rates of some pathogens (Pseudomonas aeruginosa, Candida albicans, Stenotrophomonas maltophilia and Burkholderia cepacia) were higher in ABPA patients than in those without ABPA. No association was evident in the proportion of patients with cultures positive for Staphylococcus aureus, or normal flora/negative cultures (other pathogens are extremely rare), whereas Haemophilus influenzae was reported more frequently in the non-ABPA population.

The most common respiratory complications/events found were massive haemoptysis and pneumothorax. These clinical conditions were clearly associated with ABPA. In contrast, ABPA was no more prevalent in patients who underwent lung transplantation than those in the general CF population (table 4).

Patients with ABPA received more respiratory therapies than those without ABPA, in particular, oral and inhaled corticosteroids, but also bronchodilators, dornase alfa (Pulmozyme $(\mathbb{R})$ and continuous antibiotic therapy (table 5). Conversely, ABPA was more prevalent in patients treated routinely with continuous antibiotic therapy (804/8191; $11.0 \%$ ) than in those receiving intermittent antibiotics $(5.7 \%)$ or antibiotics for exacerbations only $(3.6 \%)$. Only $26.2 \%$ of ABPA patients $(253 / 967)$ were treated with the anti-Aspergillus antibiotics, itraconazole or amphotericin (1.4\% of those without ABPA were also treated with these antibiotics).

Influence of allergic bronchopulmonary aspergillosis on lung function decline

Regression analysis of percent pred FEV1 in ABPA and non-ABPA patients was performed using the mixed model analysis, assuming linearity, for the three severity groups stratified for age. In subgroups of patients $<6$ yrs of age, analysis was not possible due to the small number of patients with valid values. The results demonstrated a marginally more rapid decline in FEV1 by age in the nonABPA patients than in the ABPA patients in several of the subgroups analysed (fig. 1; table 6). Regression analysis of percent pred FEV1 in the ABPA and non-ABPA patients after the introduction of a quadratic term for age was able to summarize the general trends in FEV1 over the follow-up period of the study (table 6), but did not identify important differences between ABPA and nonABPA patients with respect to FEV1 decline in any severity group. Only differences for the intercept values were demonstrated, which supported the poorer lung function levels in the ABPA subpopulation already identified by cross-sectional analysis.

\section{Discussion}

This is the second extensive epidemiological study of $\mathrm{ABPA}$ in $\mathrm{CF}$ to be published recently [12]. The large

Table 2. - Prevalence of allergic bronchopulmonary aspergillosis (ABPA) by initial forced expiratory volume in one second (FEV1) and age

\begin{tabular}{|c|c|c|c|c|c|c|c|c|c|c|c|c|c|c|c|c|}
\hline \multirow{3}{*}{$\begin{array}{l}\text { Age } \\
\text { yrs }\end{array}$} & \multicolumn{2}{|c|}{$\begin{array}{c}\text { CF Population } \\
n\end{array}$} & \multicolumn{2}{|c|}{$\begin{array}{l}\text { Mean FEV1 \% } \\
(95 \% \mathrm{CI})\end{array}$} & \multicolumn{4}{|c|}{$\begin{array}{l}\text { FEV } 1 \\
<40 \%\end{array}$} & \multicolumn{4}{|c|}{$\begin{array}{c}\text { FEV1 } \\
40-70 \%\end{array}$} & \multicolumn{4}{|c|}{$\begin{array}{l}\text { FEV1 } \\
\geq 70 \%\end{array}$} \\
\hline & \multirow[t]{2}{*}{ All } & \multirow[t]{2}{*}{ ABPA } & \multirow[t]{2}{*}{$\begin{array}{l}\text { No } \\
\text { ABPA }\end{array}$} & \multirow[t]{2}{*}{ ABPA } & \multirow[t]{2}{*}{$\begin{array}{l}\text { No } \\
\text { ABPA }\end{array}$} & \multicolumn{3}{|c|}{ ABPA } & \multirow[t]{2}{*}{$\begin{array}{c}\text { No } \\
\text { ABPA }\end{array}$} & \multicolumn{3}{|c|}{ ABPA } & \multirow[t]{2}{*}{$\begin{array}{l}\text { No } \\
\text { ABPA }\end{array}$} & \multicolumn{3}{|c|}{ ABPA } \\
\hline & & & & & & Yes & $\%$ & $95 \% \mathrm{CI}$ & & Yes & $\%$ & $95 \% \mathrm{CI}$ & & Yes & $\%$ & $95 \% \mathrm{CI}$ \\
\hline$<6$ & 1015 & 42 & $\begin{array}{c}91 \\
(89.5-92.5)\end{array}$ & $\begin{array}{c}78.7 \\
(73.2-84.2)\end{array}$ & 7 & 0 & 0 & $0-41.0$ & 141 & 15 & 8.4 & 4.6 & 825 & 29 & 3.4 & $2.3-4.8$ \\
\hline $6-12$ & 3284 & 306 & $\begin{array}{c}79.8 \\
(79.0-80.6)\end{array}$ & $\begin{array}{c}70.1 \\
(68.0-72.2)\end{array}$ & 136 & 16 & 10.5 & $6.1-16.5$ & 731 & 132 & 15.3 & $13.0-17.9$ & 2111 & 158 & 7.0 & $6.0-8.1$ \\
\hline $13-17$ & 2004 & 228 & $\begin{array}{c}68.5 \\
(67.4-69.6)\end{array}$ & $\begin{array}{c}60.1 \\
(57.3-62.9)\end{array}$ & 272 & 52 & 16.1 & $12.2-20.5$ & 633 & 92 & 12.7 & $10.4-15.3$ & 871 & 84 & 8.8 & $7.1-10.8$ \\
\hline$\geq 18$ & 3326 & 331 & $\begin{array}{c}54.3 \\
(53.4-55.2)\end{array}$ & $\begin{array}{c}48.3 \\
(46.1-50.5)\end{array}$ & 1001 & 141 & 12.4 & $10.5-14.4$ & 1196 & 135 & 10.1 & 8.6-11.9 & 798 & 55 & 6.5 & $4.9-8.3$ \\
\hline Total & 9629 & 907 & $\begin{array}{c}70 \\
(69.4-70.6)\end{array}$ & $\begin{array}{c}60 \\
(58.5-61.5)\end{array}$ & 1416 & 209 & 12.9 & $11.3-14.6$ & 2701 & 372 & 12.1 & $11.0-13.3$ & 4605 & 326 & 6.6 & $5.9-7.3$ \\
\hline
\end{tabular}

Only patients with initial FEV1 available are included. CF: cystic fibrosis; CI: confidence interval. 
Table 3. - The association of specific microbial infections with allergic bronchopulmonary aspergillosis (ABPA)

\begin{tabular}{lccc}
\hline Organisms & $\mathrm{n} / \mathrm{N}$ & $\mathrm{n} / \mathrm{N} \%$ & $95 \% \mathrm{CI}$ \\
\hline P. aeruginosa & & & \\
No ABPA & $6975 / 10886$ & 64.1 & $63.2-65.0$ \\
ABPA & $806 / 954$ & 84.5 & $82.0-86.7$ \\
S. aureus & & & \\
No ABPA & $6551 / 10886$ & 60.2 & $59.3-61.1$ \\
ABPA & $602 / 954$ & 63.1 & $60.0-66.2$ \\
Candida & & & \\
No ABPA & $4062 / 10886$ & 37.3 & $36.4-38.2$ \\
ABPA & $483 / 954$ & 50.6 & $47.4-53.8$ \\
Aspergillus & & & \\
No ABPA & $1764 / 10886$ & 16.2 & $15.5-16.9$ \\
ABPA & $429 / 954$ & 45.0 & $41.8-48.2$ \\
H. influenzae & & & \\
No ABPA & $4425 / 10886$ & 40.6 & $39.7-41.6$ \\
ABPA & $295 / 954$ & 30.9 & $28.0-34.0$ \\
S. maltophilia & & & \\
No ABPA & $798 / 10886$ & 7.3 & $6.8-7.8$ \\
ABPA & $159 / 954$ & 16.7 & $14.4-19.2$ \\
B. cepacia & & & \\
No ABPA & $620 / 10886$ & 5.7 & $5.3-6.1$ \\
ABPA & $75 / 954$ & 7.9 & $6.2-9.8$ \\
Other/normal flora & & & \\
No ABPA & $5329 / 10886$ & 49.0 & $48.0-49.9$ \\
ABPA & $459 / 954$ & 48.1 & 51.3 \\
\hline Oring & & & \\
\hline
\end{tabular}

Other/normal flora refers to all patients with at least one sputum culture from which rare pathogens, normal flora or no bacteria were isolated. $\mathrm{n} / \mathrm{N}$ : number of ABPA positive patients where bacteria were isolated/number of patients where bacteriology cultures were performed; $n / \mathrm{N} \%$ : proportion of $n / \mathrm{N}$ expressed as per cent; 95\% CI: 95\% confidence interval; P. aeruginosa: Pseudomonas aeruginosa; $S$. aureus: Staphylococcus aureus; $H$. influenzae: Haemophilus influenzae; S. maltophilia: Stenotrophomonas maltophilia; B. cepacia: Burkholderia cepacia.

number of patients included in this study $(>12,000)$ permitted the objective description of some epidemiological and clinical aspects of ABPA, without the need for statistical tests to evaluate the results of the descriptive analysis. Although the reliability of the parameters obtained (mean or frequencies) has been quoted as the 95\% confidence intervals for each variable analysed, the breadth of these confidence intervals is largely dependent on the number of observations per group.

\section{Allergic bronchopulmonary aspergillosis prevalence}

An overall sex-independent prevalence of ABPA in CF of $7.8 \%$ was found. Previous reports of prevalence were highly variable $[7-11,13,14]$, however, the present results cannot be compared with those of others due to the small population samples examined in these previous studies. A very low prevalence $(2 \%)$ was reported in a North American study [12], which is comparable to the present study in population size $(14,210)$. However, that prevalence was based only on medical conditions that were present within 6 months of the enrolment date. The authors of that study expressed their reservations about accepting such a low prevalence as the true prevalence of ABPA in CF and suspect that this condition is underdiagnosed for many reasons in North America.
Table 4. - Prevalence of allergic bronchopulmonary aspergillosis (ABPA) by respiratory complications

\begin{tabular}{lccr}
\hline Medical history & $\mathrm{n} / \mathrm{N}$ & $\mathrm{n} / \mathrm{N} \%$ & \multicolumn{1}{c}{$95 \% \mathrm{CI}$} \\
\hline Haemoptysis & $105 / 684$ & 15.4 & $12.7-18.3$ \\
Pneumothorax & $33 / 256$ & 12.9 & $9.0-17.6$ \\
Lung transplant & $19 / 216$ & 8.8 & $5.4-13.4$ \\
\hline
\end{tabular}

$\mathrm{n} / \mathrm{N}$ : the number of patients with ABPA affected by the specified complication/number of patients in the given class; $\mathrm{n} / \mathrm{N} \%$ : proportion of $n / \mathrm{N}$ expressed as percent; $95 \% \mathrm{CI}$ : extremes of the $95 \%$ confidence interval expressed as per cent.

The problem of variability clearly arose in the present study, as well as in the parallel epidemiological study carried out in North America [12], with prevalence ranging from $2-13 \%$ among countries and from $0-27 \%$ among CF centres. This wide variability may be partly due to the different number of cases enrolled by different centres or countries, as shown by the differences in the confidence intervals among them. Another explanation might be different levels of air-borne spores of Aspergillus spp., in different environments or geographical areas. However, it is recognized that Aspergillus spores are distributed ubiquitously in the environment [28] and that $A$. fumigatus is similarly found in all domestic environments, including hospital ventilation systems, mattress dust, between false ceilings and on plants [29]. Furthermore, it is not Aspergillus spores but soluble Aspergillus allergens that are responsible for disease: Asp $f 1$ antigen is considered the most common inciting agent in ABPA and is produced only in the mycelial phase of Aspergillus growth. While germination of spores occurs in the bronchial mucus of CF patients [30], it is unlikely in the inanimate environment. Thus, this explanation appears invalid despite the absence of evidence to the contrary.

Another reason for variability could be different $\mathrm{CF}$ management practices and differences in the overall clinical condition of patients among centres, which may lead to different levels of colonization, germination of Aspergillus spores and Aspergillus sensitization. Indeed, differences in management strategy seem to be of great importance for overall clinical condition (H.K. Harms, Childrens Hospital, Munich University, Munich, Germany, personal communication based on ERCF data). If treatment policy and disease severity correlate with ABPA development, intercentre differences in ABPA prevalence could be partly attributed to different approaches to disease management and the resulting clinical condition of patients in their care. However, it remains possible that variable interpretation of clinical and laboratory criteria in the definition of ABPA could explain, at least in part, the wide variability observed in the present study, given that ABPA diagnosis defined by the ERCF is partially based on the physician's suspicion that the condition is present.

The diagnosis of ABPA in CF remains difficult due to the commonly reported sensitization to Aspergillus in CF $[10,13,14,21,23]$, and the poor specificity of the clinical manifestations such as bronchoconstriction, central bronchiectasis and pulmonary infiltrates [31]. The suggested combination of specific immunological criteria and a four-fold rise in total $\mathrm{IgE}$, in conjunction with clinical deterioration and the newly-reported appearance of radiological shadowing in patients poorly responding 
Table 5. - Routine respiratory therapy rate in allergic bronchopulmonary aspergillosis (ABPA) and non-ABPA patients

\begin{tabular}{lccc}
\hline & $\mathrm{n} / \mathrm{N}$ & $\mathrm{n} / \mathrm{N} \%$ & $95 \% \mathrm{CI}$ \\
\hline Bronchodilators & & & \\
No ABPA & $7726 / 11480$ & 67.3 & $66.4-68.2$ \\
ABPA & $876 / 967$ & 90.6 & $88.6-92.4$ \\
Corticosteroid (inhaled) & & & \\
No ABPA & $4432 / 11480$ & 38.6 & $37.7-39.5$ \\
ABPA & $725 / 967$ & 75.0 & $72.1-77.7$ \\
Corticosteroid (oral) & $1693 / 11480$ & 14.7 & $14.1-15.4$ \\
No ABPA & $543 / 967$ & 56.2 & $53.0-59.3$ \\
ABPA & & & \\
Dornase alfa & $4523 / 11480$ & 39.4 & $38.5-40.3$ \\
No ABPA & $628 / 967$ & 64.9 & $61.8-68.0$ \\
ABPA & & & \\
Antibiotics (continuous) & $7387 / 11480$ & 64.3 & $63.5-65.2$ \\
No ABPA & $804 / 967$ & 83.1 & $80.6-85.5$ \\
ABPA & & & \\
Antibiotics (intermittent) & $1316 / 11480$ & 11.5 & $10.9-12.1$ \\
No ABPA & $79 / 967$ & 8.2 & $6.5-10.1$ \\
ABPA & $1558 / 11480$ & 13.6 & $12.9-14.2$ \\
Antibiotics (exacerbations) & $58 / 967$ & 6.0 & $4.6-7.7$ \\
No ABPA & & & \\
ABPA &
\end{tabular}

$\mathrm{n} / \mathrm{N}$ : the number of patients using the given treatment/the number of patients in the given class; $n / \mathrm{N} \%$ : proportion of $n / \mathrm{N}$ expressed as per cent; $95 \%$ CI: $95 \%$ confidence interval of the proportion $\mathrm{n} / \mathrm{N}$ expressed as per cent.

to antibiotics [20], may improve the diagnosis of ABPA in CF. Elevated serum IgE in A. fumigatus-sensitized patients has been claimed by others to be clinically relevant [23]. At present, one of the major problems in diagnosing ABPA arises from the lack of standardized fungal extracts [32]. A new diagnostic method has been proposed based on particular disease-specific recombinant $A$. fumigatus allergens $[32,33]$. Two categories of $A$. fumigatus allergens have been identified: secreted and cytoplasmic proteins. Secreted allergens may be recognized by serum IgE of $A$. fumigatus-sensitized individuals with or without ABPA, whereas nonsecreted cytoplasmic allergens may be recognized only by serum IgE from ABPA patients [32]. By using such allergens, distinct IgE responses to $A$. fumigatus have been found in $\mathrm{CF}$ patients with ABPA (against $r A s p f 4$ and $r A s p f 6$ antigens) or simple $A$. fumigatus allergy (against $r A s p F 1$ and $r A s p F 3$ antigens) $[22,34])$. Differentiation of the two conditions is fundamental because of different therapeutic implications.

There are only a limited number of reports in the literature that have addressed the prevalence of ABPA at an early age in $\mathrm{CF}$, despite evidence that an immune response to A. fumigatus usually develops very early in these patients $[10,12,21]$. Our data show that ABPA can affect even patients $<6$ yrs of age (56/3150 patients). In patients $\geq 6$ yrs of age, prevalence is consistently $\sim 10 \%$.

An attempt to correlate the prevalence of ABPA phenotype with CFTR genotype was performed by grouping patients with a known genotype into the four most common groups found among the ERCF patients $(\triangle \mathrm{F} 508 /$ $\Delta \mathrm{F} 508, \Delta \mathrm{F} 508 / \mathrm{G} 55 \mathrm{ID}, \Delta \mathrm{F} 508 / \mathrm{G} 542 \mathrm{X}, \Delta \mathrm{F} 508 / \mathrm{N} 1303 \mathrm{~K})$. The remaining patients were grouped into two classes: $\Delta \mathrm{F} 508 /$ other and other/other. No significant differences were observed between genotypes.

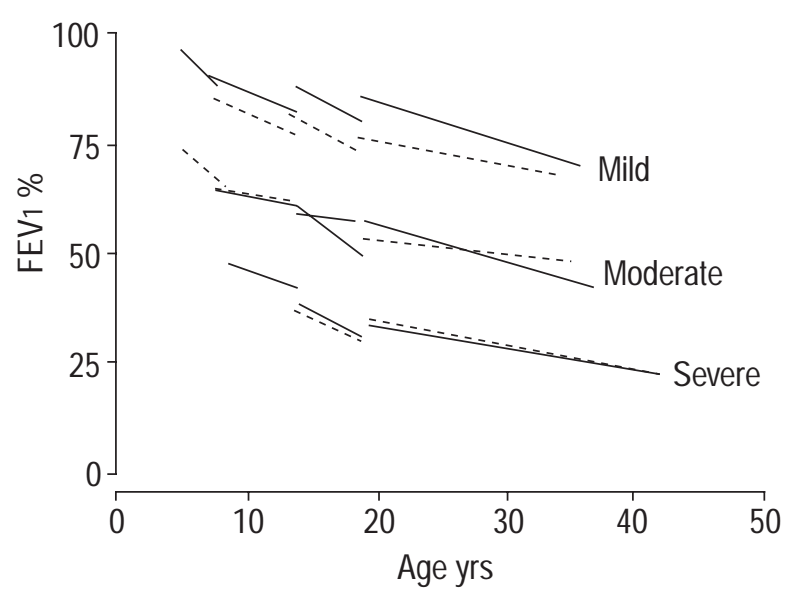

Fig. 1. - Mixed model regression lines of forced expiratory volume in one second (FEV1) (\%) versus age (yrs) for ABPA ( - - ) and nonABPA ( - ) patients. The dates are divided into three disease severity categories determined on the basis of FEV1 at enrolment, and each severity category is subdivided into four age classes at enrolment (from left to right: $<6$ yrs, 6-12 yrs, 13-17 yrs, $\geq 18$ yrs.)

\section{Allergic bronchopulmonary aspergillosis associations with clinical conditions}

ABPA was generally associated with a poorer nutritional status (lower body weight Z-score), slightly higher concentrations of serum IgG (an indication of greater inflammation), a poorer respiratory function (lower FEV1 and FVC) and a higher rate of respiratory tract colonization by pathogens.

Association with impaired lung function is of interest. Patients with ABPA had, on average, a 10\% lower FEV1 \% pred than those without ABPA. In addition, patients with FEV $1 \geq 70 \%$ of the predicted value were less frequently affected by ABPA (7\%) than the others, while in patients with FEV $1<70 \%$ of the predicted value ABPA can reach peak frequencies of $13-16 \%$ in adolescents (table 2). Poorer lung function in CF patients with ABPA or sensitization to A. fumigatus has been reported $[10,23]$. In $\mathrm{CF}$ it is unclear whether ABPA is responsible for the deterioration, more severe lung impairment predisposes patients to ABPA, or some other clinical feature underlies both conditions. The absence of complete clinical and immunological patient histories prior to their enrolment in the ERCF means that these data cannot answer this question.

A greater frequency of pathogenic bacterial colonization of the bronchial tree in CF patients with ABPA is a novel observation. ABPA patients are more frequently colonized by $P$. aeruginosa, S. maltophilia, B. cepacia and $C$. albicans as well as $A$. fumigatus than those without ABPA. Again the causal relationship between these observations is unclear. In contrast, colonization by $S$. aureus was comparable between subgroups, probably due to its colonization of the youngest patients. The young age could also account for the greater colonization by $H$. influenzae of the non-ABPA subjects. It is important to reiterate that only a proportion of ABPA patients show a sputum culture positive for A. fumigatus.

The observation that ABPA patients have more severe pulmonary disease than those without ABPA is consistent with the higher incidence of lung complications such as 
Table 6. - Forced expiratory volume in one second (FEV1) percentage decline by age according to initial severity groups independent of the age at enrolment (mixed model analysis)

\begin{tabular}{|c|c|c|c|c|c|c|c|}
\hline \multirow[b]{2}{*}{ Severity group } & \multirow{2}{*}{ Population $n$} & \multicolumn{3}{|c|}{ Slopes FEV1 \% } & \multicolumn{3}{|c|}{ p-values $^{\#}$} \\
\hline & & Intercept & Linear & Quadratic & Intercept & Linear & Quadratic \\
\hline \multicolumn{8}{|l|}{ Severe } \\
\hline non-ABPA & 1072 & $69.3 \pm 2.7$ & $-2.7 \pm 0.3$ & $0.04 \pm 0.01$ & & & \\
\hline ABPA & 167 & $57.5 \pm 5.5$ & $-1.6 \pm 0.5$ & $0.02 \pm 0.01$ & 0.06 & 0.06 & 0.08 \\
\hline \multicolumn{8}{|l|}{ Moderate } \\
\hline non-ABPA & 2035 & $79.8 \pm 1.4$ & $-1.8 \pm 0.2$ & $0.03 \pm 0.00$ & & & \\
\hline ABPA & 306 & $71.1 \pm 3.7$ & $-0.9 \pm 0.4$ & $0.01 \pm 0.01$ & 0.03 & 0.06 & 0.13 \\
\hline \multicolumn{8}{|l|}{ Mild } \\
\hline non-ABPA & 3249 & $101.6 \pm 1.1$ & $-1.5 \pm 0.2$ & $0.02 \pm 0.00$ & & & \\
\hline ABPA & 258 & $91.6 \pm 4.8$ & $-1.0 \pm 0.6$ & $0.01 \pm 0.02$ & 0.04 & 0.43 & 0.46 \\
\hline
\end{tabular}

Data represents mean \pm SEM. ABPA: allergic bronchopulmonary aspergillosis; ${ }^{\#}$ : ABPA versus non-ABPA.

massive haemoptysis and pneumothorax in those with ABPA. Lung transplantation was performed in a similar proportion of patients in the two subpopulations.

The poorer pulmonary status of ABPA patients is also consistent with the more intensive and aggressive therapy administered. All of the common therapies used to treat lung disease in $\mathrm{CF}$ were administered to considerably more ABPA patients than those without ABPA. Orally-administered corticosteroids are generally recommended for ABPA in non-CF patients [27] as well as in CF patients [11, 16, $18,19]$ in order to control acute exacerbations and prevent permanent lung damage. Notably, only $56 \%$ of the ABPA patients received oral corticosteroids while $75 \%$ of them received inhaled corticosteroids. However, the latter route of administration was also commonly used in the non-ABPA patients (39\%). There has been no evidence to date of the effectiveness of inhaled corticosteroids in controlling ABPA in $\mathrm{CF}$. The greater use of continuous antibiotics and dornase alfa in patients with ABPA probably confirms the greater severity of lung disease in these patients, but one could also argue that continuous exposure to antibiotics may predispose to fungal colonization.

\section{Influence of allergic bronchopulmonary aspergillosis on lung function decline}

Despite the relatively short period of follow-up, the longitudinal aspect of this study has provided some interesting insight into independent effects of ABPA on the progression of pulmonary disease in CF. In the crosssectional part of the present study, it has been demonstrated that ABPA is generally associated with a poorer pulmonary status, although a significant causal relationship is yet to be established. This is partly supported by other studies $[10$, $12,23]$.

The mixed model regression analysis was unable to demonstrate substantial or clinically relevant differences in the rates of FEV1 decline between ABPA and non-ABPA patients for any of the age by severity subgroups considered. In some subgroups, typically in the adult group with severe impairment, the regression lines are indistinguishable, whereas in other subgroups, including the adolescent and adult groups with moderate lung function impairment, FEV1 decline seems to be more rapid in the patients without ABPA. The results of this analysis are not conclusive and a longer period of observation, combined with the evaluation of other variables involved in the decline of pulmonary function, is necessary.

In conclusion, this study enabled the authors to estimate the variable prevalence of allergic bronchopulmonary aspergillosis in cystic fibrosis in countries of western, central and northern Europe. It has been demonstrated that this complication is associated with a poorer clinical condition. A longitudinal approach using a mixed model multiple regression analysis was unable to confirm an independent contribution of allergic bronchopulmonary aspergillosis to the progress of lung disease in the short term.

\footnotetext{
Acknowledgements. The authors gratefully acknowledge the assistance and contribution of the Epidemiologic Registry of Cystic Fibrosis (ERCF) coordinators and investigators.
}

\section{References}

1. McCarthy DS, Pepis J, Allergic bronchopulmonary aspergillosis. 1: Clinical features. Clin Allergy 1971; 1: 261284.

2. Greenberger PA. Allergic bronchopulmonary aspergillosis. In: Middleton JE, et al. eds, Principles and Practice, Vol. II, 4th Ed. St Louis, MO, Mosby, 1993; 1395-1414.

3. Greenberger PA. Allergic bronchopulmonary aspergillosis. In: Patterson R, ed, Allergic Diseases, Diagnosis and Management, 4th Ed. Philadelphia, PA, JP Lippincott Co, 1993; 763-787.

4. Slavin RG, Bedrossian CW, Hutcheson PS, et al. A pathological study of allergic bronchopulmonary aspergillosis. J Allergy Clin Immunol 1988; 81: 718-725.

5. Nelson LA, Callerame ML, Schwartz RH. Aspergillosis and atopy in cystic fibrosis. Am Rev Resp Dis 1979; 120: 863-873.

6. Robinson BNS, Venaille TJ, Mendls AHW, McAleer R. Allergens as proteinases: an Aspergillus fumigatus proteinase directly induces human epithelial cell detachment. J Allergy Clin Immunol 1990; 86: 726-731.

7. Feanny S, Forsyth S, Corey M, Levison H, Zimmerman B. Allergic bronchopulmonary aspergillosis in cystic fibrosis: a secretory immune response to a colonizing organism. Ann Allergy 1988; 60: 64-68. 
8. Laufer P, Fink TN, Bruns TH, et al. Allergic bronchopulmonary aspergillosis in cystic fibrosis. $J$ Allergy Clin Immunol 1984; 73: 44-48.

9. Simmonds EJ, Littlewood JM, Evans EGV. Cystic fibrosis and allergic bronchopulmonary aspergillosis. Arch Dis Child 1990; 65: 507-511.

10. Valletta E, Braggion C, Mastella G. Sensitization to Aspergillus and allergic bronchopulmonary aspergillosis in a cystic fibrosis population. Pediatr Asthma Allergy Immunol 1993; 7: 43-49.

11. Mroueh S, Spock A. Allergic bronchopulmonary aspergillosis in patients with cystic fibrosis. Chest 1994; 105: 32-36.

12. Geller DE, Kaplowitz H, Light MJ, Colin A. Allergic bronchopulmonary aspergillosis in cystic fibrosis. Reported prevalence, regional distribution, and patient characteristics. Chest 1999; 116: 639-646.

13. Becker JW, Burke W, McDonald G, Greenberger PA, Henderson WR, Aitken ML. Prevalence of allergic bronchopulmonary aspergillosis and atopy in adult patients with cystic fibrosis. Chest 1996; 109: 1536-1540.

14. Hutcheson PS, Knudson AP, Rejent AJ, Slavin RG. A 12year longitudinal study of Aspergillus sensitivity in patients with cystic fibrosis. Chest 1996; 110: 363-366.

15. Zhaomin W, Lockey RF. A review of allergic bronchopulmonary aspergillosis. Invest Allergy Clin Immunol 1996; 6: 144-151.

16. Slavin RG. ABPA in CF: a devastating combination. Pediatr Pulmonol 1996; 21: 1-2.

17. Brueton MJ, Ormerod LP, Shall KJ, Anderson LM. Allergic bronchopulmonary aspergillosis complicating cystic fibrosis in childhood. Arch Dis Child 1980; 55: 348-353.

18. Voss MJ, Bush RK, Mischler E, Peters ME. Association of allergic bronchopulmonary aspergillosis and cystic fibrosis. J Allergy Clin Immunol 1982; 69: 539-546.

19. Kolls JK, Beckerman RC. Will ABPA occur in your patient with cystic fibrosis? J Respir Dis 1991; 12: 137-146.

20. Marchant JL, Warner JO, Bush A. Rise in total IgE an indicator of allergic bronchopulmonary aspergillosis in cystic fibrosis. Thorax 1994; 49: 1002-1005.

21. El-Dahr JM, Fink R, Selden R, Arruda LK, Platts-Mills TAE, Heymann PW. Development of immune responses to Aspergillus at an early age in children with cystic fibrosis. Am J Respir Crit Care Med 1994; 150: 1513-1518.
22. Nikolaizik WH, Crameri R. Blaser K, Schöni MH. Skin test reactivity to recombinant Aspergillus fumigatus allergen I/a in patients with cystic fibrosis. Int Arch Allergy Immunol 1996; 111: 403-408.

23. Wojnarowski C, Eichler I, Gartner C, et al. Sensitization to Aspergillus fumigatus and lung function in children with cystic fibrosis. Am J Respir Crit Care Med 1997; 155: 1902-1907.

24. Knudson RJ, Lebowitz MD, Holberg CJ, Burrows B. Changes in the normal maximal expiratory flow-volume curve with growth and aging. Am Rev Resp Dis 1983; 127: 725-734.

25. Dean AG, Dean GA, Burton AH, Dicker RG. EPI INFO: Version 5: A word processing, database and statistic program for epidemiology or microcomputer. Stone Mountain, Georgia, USD inc. 1990.

26. Lairo NM, Ware JA. Random-effects models for longitudinal data. Biometrics 1982; 38: 963-974.

27. Coley M, Edwards L, Levison H, Knowles M. Longitudinal analysis of pulmonary function decline in patients with cystic fibrosis. J Pediatr 1997; 131: 809-814.

28. Bardana EJ. The clinical spectrum of aspergillosis. 2. Epidemiology, pathogenicity, infection in animals and immunology. CRC Crit Rev Clin Lab Sci 1980; 13: 21-84.

29. Prahl P. Reduction of indoor mould spores. Allergy 1992; 47: 362-365.

30. Arruda LK, Platts-Mills AE, Fox JW, Chapman MD. Aspergillus fumigatus allergen I, is a member of the mitogillin family of cytotoxins. $J$ Exp Med 1990; 172: $1529-1532$.

31. Greenberger PA, Patterson R. Diagnosis and management of allergic bronchopulmonary aspergillosis. Ann Allergy 1986; 56: 444-448.

32. Crameri R. Recombinant Aspergillus fumigatus allergens: from the nucleotide sequences to clinical applications. Int Arch Allergy Immunol 1998; 115: 99-114.

33. Crameri R, Hemmann S, Ismail C, Menz G, Blaser K. Disease-specific recombinant allergens for the diagnosis of allergic bronchopulmonary aspergillosis. Int Immunol 1998; 10: 1211-1216.

34. Hemmann S, Nikolaizik WH, Schöni MH, Blaser K, Crameri R. Differential IgE recognition of recombinant Aspergillus fumigatus allergens by cystic fibrosis patients with allergic bronchopulmonary aspergillosis or Aspergillus allergy. Eur J Immunol 1998; 28: 1155-1160. 